\title{
Intra-tumoral susceptibility signal: a post-processing technique for objective grading of astrocytoma with susceptibility-weighted imaging
}

\author{
Tzu-Chao Chuang ${ }^{1}$, Yen-Lin Chen ${ }^{1}$, Wan-Pin Shui ${ }^{1}$, Hsiao-Wen Chung ${ }^{2}$, Shu-Shong Hsu ${ }^{3}$, Ping-Hong Lai ${ }^{4,5}$ \\ ${ }^{1}$ Department of Electrical Engineering, National Sun Yat-sen University, Kaohsiung; ${ }^{2}$ Department of Electrical Engineering, National Taiwan \\ University, Taipei; ${ }^{3}$ Department of Neurosurgery, Kaohsiung Veterans General Hospital, Kaohsiung; ${ }^{4}$ Department of Radiology, Kaohsiung Veterans \\ General Hospital, Kaohsiung; ${ }^{5}$ School of Medicine, National Yang Ming Chiao Tung University, Taipei
}

Contributions: (I) Conception and design: TC Chuang, PH Lai; (II) Administrative support: TC Chuang, PH Lai; (III) Provision of study materials or patients: SS Hsu, PH Lai; (IV) Collection and assembly of data: PH Lai; (V) Data analysis and interpretation: TC Chuang, YL Chen, WP Shui; (VI) Manuscript writing: All authors; (VII) Final approval of manuscript: All authors.

Correspondence to: Ping-Hong Lai, MD. Department of Radiology, Kaohsiung Veterans General Hospital, 386 Ta-Chung First Rd., Kaohsiung.

Email: pinghonglai@gmail.com.

Background: Susceptibility-weighted imaging (SWI) is sensitive to the accumulation of paramagnetic substances, such as hemorrhage and increased venous vasculature, both being frequently found in high-grade tumors. The purpose of this retrospective study is to differentiate high-grade and low-grade astrocytoma by objectively measuring quantitative intra-tumoral susceptibility signals (qITSS) on SWI.

Methods: Precontrast SWI and 3D contrast-enhanced T1WI of 65 patients with astrocytoma were collected at 1.5 Tesla. All tumors were histologically confirmed and classified into two groups: high grade (WHO grade III and IV, n=50) and low grade (WHO grade II, n=15). After manual delineation of the tumor on T1WI, normalized contrast (NC) was calculated voxel by voxel within the tumor by using the concept of contrast to noise ratio. Thresholding on NC was applied to detect qITSS, and the volumetric percentage of qITSS can be obtained for each tumor. Two-sample $t$-test was applied to examine significant difference of qITSS percentage between high-grade and low-grade astrocytoma for different NC thresholds, ranging from 4 to 20. Receiver operating characteristic analysis was performed to evaluate the performance of differentiation.

Results: P value was less than 0.01 for a large range of NC thresholds [4-20], reflecting significant difference of qITSS percentage between high-grade and low-grade astrocytoma. The area under the receiver operating characteristic curve was larger than 0.9 at NC thresholds from 8 to 16 and peaks at 0.949 with a NC threshold of 14. It was shown that astrocytoma grading by qITSS percentage is successful for a wide range of NC threshold, demonstrating robustness on threshold selection.

Conclusions: Without relying on the selection of slice position and the same time providing objective identification of hypointense signal in SWI, the qITSS percentage can be used to distinguish high-grade and low-grade astrocytoma reliably.

Keywords: Susceptibility-weighted imaging (SWI); astrocytoma; tumor grading

Submitted Jan 14, 2021. Accepted for publication May 26, 2021.

doi: 10.21037/qims-21-58

View this article at: https://dx.doi.org/10.21037/qims-21-58 


\section{Introduction}

To better visualize the magnetic susceptibility differences of several important biological tissues, such as venous blood vessels and iron-laden tissues, susceptibility-weighted imaging (SWI) has been developed to collect data by a three-dimensional, fully velocity-compensated, long-echotime gradient echo sequence and combine the magnitude and phase in image reconstruction $(1,2)$. In general, the paramagnetic tissues with distinct susceptibility relative to their surroundings, such as hemorrhage, would appear as hypointensity in SWI. Therefore, it has become an emerging technique in neuroimaging to reveal the abnormalities of vasculature and microbleeds (3).

Aggressive tumors tend to exhibit angiogenesis and microhemorrhage, and as a result, tumor characterization can benefit from the identification of increased vasculature and intratumoral hemorrhage using SWI (4). Many studies have reported that the appearance of hypointense signals with a fine linear- or dot-like structure in brain tumors, which is termed as intra-tumoral susceptibility signal (ITSS), helps either differentiation of various types of brain tumors (5-11) or tumor grading (12-17). Among these retrospective studies, the degree of ITSS was mostly defined by visual assessment with a qualitative scale (i.e., no appearance, low, medium, and high frequency) $(5,9,14)$, the size (14) or the number (13) of ITSS, or a semi-quantitative score $(6,7,10$ $12,15,16)$. For the last approach, an ITSS score of 0 to 3 $(7,11,12,15-17)$ or 1 to $3(6,10)$ is evaluated according to the number of the ITSS on a single selected slice, usually axial, that visually shows the maximum ITSS.

Although different strategies were mentioned above, the concept to quantify the presence of ITSS in SWI using these documented approaches is similar. However, the previous proposed ITSS assessment might be influenced by observation conditions and has difficulty in reproducibility due to two reasons. First, the number of hypointense dots or tubular structures may depend on the slice location and orientation because the distribution of increased vasculature and microhemorrhage might not be uniform around the tumor. Even the thickness of slice has a direct effect on the ITSS. The use of minimum intensity projection across the whole tumor (13) might help avoid the slice dependency, but the contamination from outside of the tumor could occur and is impossible for correction. Another problem is that subjective categorization becomes inevitable when the presence and number of ITSS needs to be determined by visual inspection. A semi-automatic segmentation has thus been proposed to determine the ITSS using simple thresholding (8). According to its definition, those voxels with signal intensity lower than the median value of a reference region placed in the ventricular system were classified as the ITSS, followed by the calculation of ITSS volume and its percentage of the tumor. However, the signal of cerebrospinal fluid, which has much longer T1 and T2 relaxation times than brain parenchyma, would depend on the setting of scanning parameters (especially TE, TR and flip angle) and the magnetic field strength. In other words, this simple thresholding may encounter obstacles with different imaging protocols. As a consequence, the purpose of our study is to establish an objective and quantitative measurement of ITSS for grading brain astrocytoma, and to evaluate the robustness of the proposed method upon high- and low-grade differentiation at various thresholding parameter settings.

\section{Methods}

\section{Subjects}

A series of 65 patients with astrocytic gliomas was enrolled in this study. Final pathology results of all subjects were obtained from surgically removed specimens. Astrocytic gliomas were identified and graded according to the standards described by the 2007 World Health Organization (WHO) classification of the central nervous system tumors (18) and sorted into two groups: low-grade (grade II) and high-grade (grade III and grade IV) groups. Of the total 65 tumors (39 males and 26 females; $13-88$ years old), histopathological diagnoses were glioblastomas $(n=45, W H O$ grade IV), anaplastic astrocytoma $(\mathrm{n}=5, \mathrm{WHO}$ grade III), and lowgrade astrocytoma $(\mathrm{n}=15$, WHO grade II). The study was conducted in accordance with the Declaration of Helsinki (as revised in 2013). The in vivo study was approved by the institutional review board of Kaohsiung Veterans General Hospital, and informed consents were obtained from all patients.

\section{MR imaging}

All patients underwent SWI and conventional MR imaging, including T1WI, T2WI, and T2-FLAIR on a 1.5-T scanner (Signa HDx, GE Healthcare, Milwaukee, WI). To obtain SWI, a 3D flow-compensated gradient-echo sequence was applied with TE/TR $=39 / 50 \mathrm{~ms}$, flip angle $=18^{\circ}$, FOV $=22 \mathrm{~cm}$, acquisition matrix $=288 \times 256 \times 28$, readout 
bandwidth $=61 \mathrm{~Hz} /$ pixel, and slice thickness $=2.5 \mathrm{~mm}$. For the delineation of the tumor, 3D contrast-enhanced (CE) T1WI of the whole brain was performed by using inversion prepared spoiled gradient echo sequence with TE/TR/TI $=4 \cdot 2 / 9.2 / 400 \mathrm{~ms}$, flip angle $=20^{\circ}, \mathrm{FOV}=24 \mathrm{~cm}$, acquisition matrix $=288 \times 160 \times 124$, bandwidth $=122 \mathrm{~Hz} /$ pixel, and slice thickness $=1.2 \mathrm{~mm}$. In addition, multi-slice transverse T2FLAIR was also collected by using fast spin-echo with the following parameters: TE/TI/TR $=128 / 2,200 / 9,000 \mathrm{~ms}$, FOV $=24 \mathrm{~cm}$, acquisition matrix $=256 \times 192$, and slice thickness $=5 \mathrm{~mm}$.

\section{Image analysis}

The image processing of SWI was conducted offline according to the procedure described in the literature $(2,19)$. First, to eliminate the interference of the background field, a 2D high-pass Gaussian filter with a full-width at halfmaximum of 32 cycles per FOV was applied on the k-space. Then the high-pass filtered phase image was normalized to produce the phase mask. To generate SWI, the original magnitude image in axial view was multiplied by the phase mask four times, which is typically used. Finally, each SWI was reconstructed in axial orientation with a matrix size of $512 \times 512$ in this study. All image processing software customized to this study, including delineation of the tumor to be described later, was developed on Matlab (MathWorks, v9.0, RRID:SCR_001622).

To determine the volume and percentage of quantitative intra-tumoral susceptibility signal (qITSS), an objective method based on SWI contrast is proposed. First, the 3D CE-T1WI was registered to the 3D SWI using a rigid-body model with maximization of normalized mutual information (20) to prevent possible movement between scans. This step was done by applying Statistical Parametric Mapping software package (SPM12). Notice that the co-registered CE-T1WI was resampled in the same resolution with SWI to prevent excessive interpolation in the slice direction. The boundary of the lesion, which usually presents contrast-enhancing contour or hypointense signal on the co-registered CE T1WI, was manually delineated through all intercepted slices. For lesions that were not clearly enhanced, delineation on CE T1WI was performed with assistance by referencing to FLAIR images. Based on the concept of contrast-to-noise ratio, the normalized contrast (NC) of SWI was calculated for each voxel (with its intensity $\mathrm{I}_{\mathrm{SWI}}$ ) within the lesion to detect the qITSS by:

$$
\mathrm{NC}=\left(\mu_{\text {non-ITSS }}-\mathrm{I}_{\mathrm{SWI}}\right) / \mathrm{STD}_{\mathrm{NWM}}
$$

where $\mu_{\text {non-ITSS }}$ indicates the mean SWI intensity of nonITSS voxels in the tumor, which can be estimated by averaging the class with higher mean after applying Otsu's thresholding (21) on the lesion. The Otsu's method is a well-known algorithm of cluster-based image thresholding, in which two groups of voxels were separated by an optimal threshold to minimize the intra-class variance. Please notice that the ITSS, previously defined as low signal intensity in fine linear or dot-like structure (12), is theoretically classified into the group with gray level below the Otsu's threshold. But not all voxels of this lower group necessarily belong to ITSS, especially in consideration of those lesions with ITSS absence. On the other hand, the higher group would contain no ITSS, so that its mean can serve as a good reference of $\mu_{\text {non-ITss. }}$ Also in Eq. [1], STD NWM $_{\text {indicates the }}$ standard deviation of a normal-appearance white matter region that was manually selected as a quadrilateral area located on the contralateral brain side of the lesion on SWI of the identical case, representing the degree of signal fluctuation. As a result, regions with low gray levels in SWI, usually suspected as hemorrhage or venous blood, tend to exhibit high $\mathrm{NC}$ values.

The volume of qITSS was therefore obtained by counting all voxels with their NC larger than a predefined threshold in the whole tumor. Since the optimal NC threshold for tumor grading is unknown, various values, ranging from 4 to 20, were explored in this study. For each threshold, a two-tailed two-sample t-test with uneven standard deviation was applied on the volumetric percentage of ITSS to examine statistical difference and hence the differentiation ability between low-grade and high-grade astrocytomas. A P value less than 0.01 was considered as significant difference. To evaluate the performance of differentiation, receiver operating characteristic (ROC) analysis was performed, and the area under the ROC curve (AUC) was calculated.

\section{Results}

Figure 1 shows an axial slice of CE T1WI (A) and SWI (B) of a 51-year male patient with a right parietal glioblastoma (WHO grade IV). The contour of the tumor is drawn by green. The region of qITSS, which is delineated in red, obtained at the NC thresholds from 8 to 16 is compared in Figure $1 C$ from left to right. By setting NC threshold as $8,10,12,14$, and 16 , the volume of qITSS of this case 

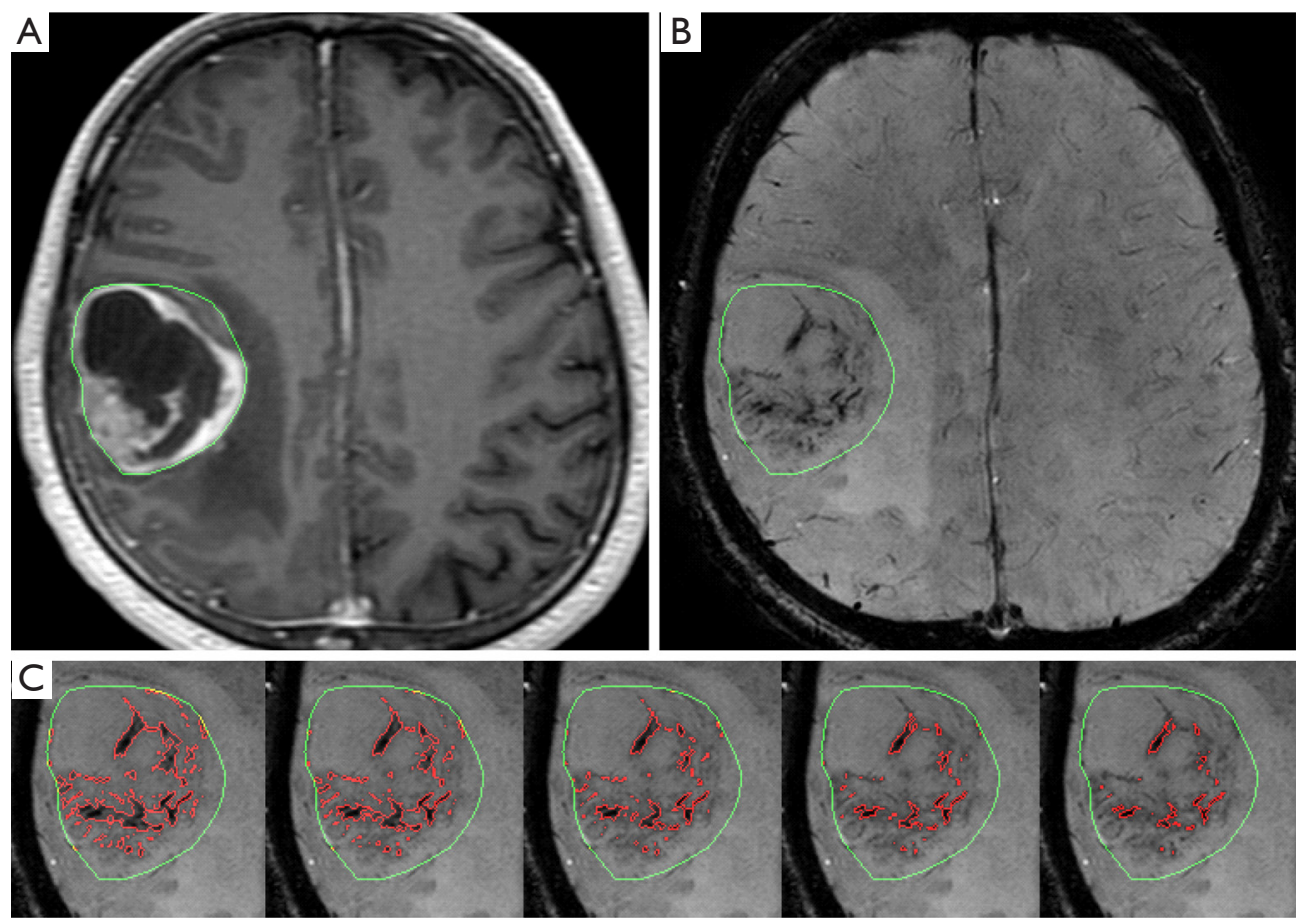

$\mathrm{NC}>8$

NC $>10$
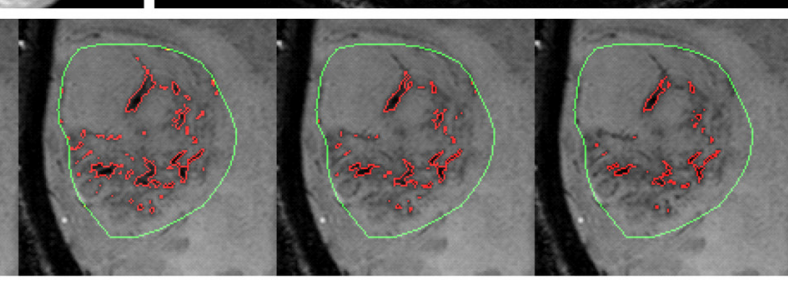

Figure 1 Cross-sectional images of a 51-year male patient with a right parietal glioblastoma (contoured in green) and the qITSS (in red) detected at different NC thresholds. (A) CE-T1WI. (B) SWI. (C) Cropped SWI to show that as the NC threshold increases from 8, 10, 12, 14 , to 16 , the region of qITSS (red contour) reduces, leading to a volumetric percentage of $17.04 \%, 11.84 \%, 8.32 \%, 5.73 \%$, and $4.00 \%$ with the total tumor volume of $43.95 \mathrm{~mL}$. qITSS, quantitative intra-tumoral susceptibility signal; NC, normalized contrast; CE T1WI, contrastenhanced T1-weighted image; SWI, susceptibility-weighted image.

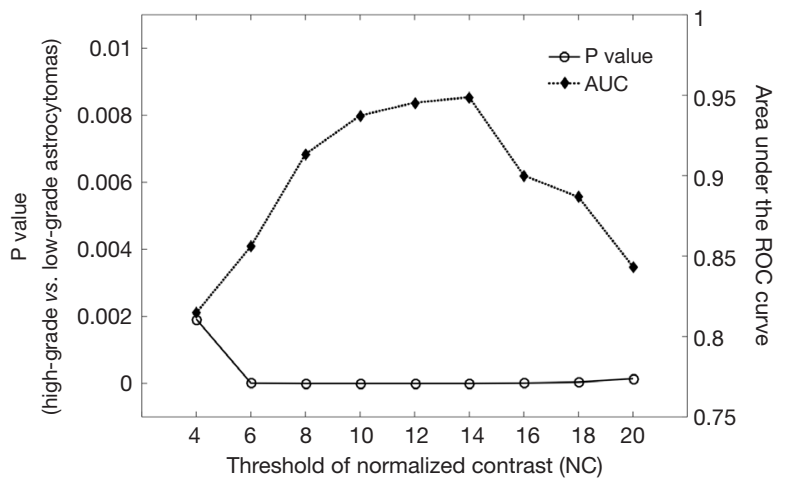

Figure 2 The p value (in circle) and AUC (in solid diamond) obtained at NC thresholds from 4 to 20. Significant difference $(\mathrm{P}<0.01)$ is achieved for all NC thresholds ranging from 4 to 20 . As the threshold increases from 4 to 6 , the $\mathrm{P}$ value drops rapidly and remains low when the threshold is larger than or equal to 6 . Meanwhile, the AUC increases gradually and reaches to a plateau at thresholds from 10 to 14 . The maximal AUC is 0.949 when the threshold is set at 14 . AUC, area under the ROC curve; NC, normalized contrast. is $7.49,5.21,3.66,2.52$, and $1.76 \mathrm{~mL}$, corresponding to a percentage of $17.04 \%, 11.84 \%, 8.32 \%, 5.73 \%$, and $4.00 \%$ with the total tumor volume of $43.95 \mathrm{~mL}$, respectively. It is obvious that as the threshold increases, less qITSS is detected.

Figure 2 demonstrates the threshold dependency plot of $\mathrm{p}$ value (solid line with circle marks) and AUC (dot line with diamond marks). For all the NC thresholds ranging from 4 to 20, significant difference is always found between highgrade and low-grade tumors $(\mathrm{P}<0.01)$, which means that the distribution of qITSS percentage of high-grade and lowgrade astrocytomas is apparently different for a wide range of NC threshold. On the other hand, the AUC is maximized as 0.949 at a $\mathrm{NC}$ threshold of 14 (Figure 2), suggesting the optimal NC threshold corresponding to an accuracy of $89 \%$ for grading of astrocytoma specifically for our patients. In fact, using a NC threshold of 14, the ITSS region is found to best match visual observation by our experienced neuroradiologists (see Figure 1). Nonetheless, it is seen that AUC values at thresholds from 10 to 14 are very close to 
Table 1 The volume of high- and low-grade astrocytomas and the qITSS measured by using NC $>14$

\begin{tabular}{lcc}
\hline & High-grade $(\mathrm{n}=50)$ & Low-grade $(\mathrm{n}=15)$ \\
\hline Volume of tumor $(\mathrm{mL})$ & & \\
Range & $1.57-163.11$ & $14.19-104.53$ \\
Mean/SD & $46.98 / 31.81$ & $45.35 / 38.60$ \\
Volume of qITSS $(\mathrm{mL})$ & & \\
Range & $0.01-36.40$ & $0.01-0.71$ \\
Mean/SD & $3.07 / 5.66$ & $0.13 / 0.24$ \\
qITSS percentage & & $0.00-0.68 \%$ \\
Range & $0.03-30.1 \%$ & $0.04 \%$ \\
Median & $3.62 \%$ & $0.15 \% / 0.23 \%$ \\
Mean/SD & $5.79 \% / 6.86 \%$ & \\
\hline
\end{tabular}

qITSS, quantitative intra-tumoral susceptibility signal.

each other, all larger than 0.93 . Hence the differentiation between low- and high-grade astrocytomas using the proposed qITSS percentage method is not sensitive to the choice of NC threshold, as long as the NC threshold falls within reasonable range. With the NC threshold set as 14 , the volume of tumor and qITSS is summarized for highgrade and low-grade tumors in Table 1, and the box plot of qITSS percentage is shown as Figure 3.

\section{Discussion}

The major advancement of this study is the quantification of the ITSS score, which changes from manual counting of hypo-intense structures on a representative $2 \mathrm{D}$ slice to automatic computation of the percentage volume ratio. Volume calculation depends on the choice of NC threshold, which has been evaluated by varying the threshold for a wide range. By using NC $>14$ for qITSS determination, significant difference between high-grade and low-grade astrocytomas is found on the volumetric percentage of ITSS, and an AUC of 0.949 was obtained, rendering this quantitative index as a helpful classifier on discrimination of tumor grade. Furthermore, a similar threshold, such as 10 or 12, can still provide good identification of qITSS and similar capability of differentiation, reaching an AUC of 0.937 and 0.945 , respectively. In other words, the differentiation is not susceptible to NC threshold, at least for tumor grading of astrocytoma conducted in this study, suggesting robustness of our volumetric approach for

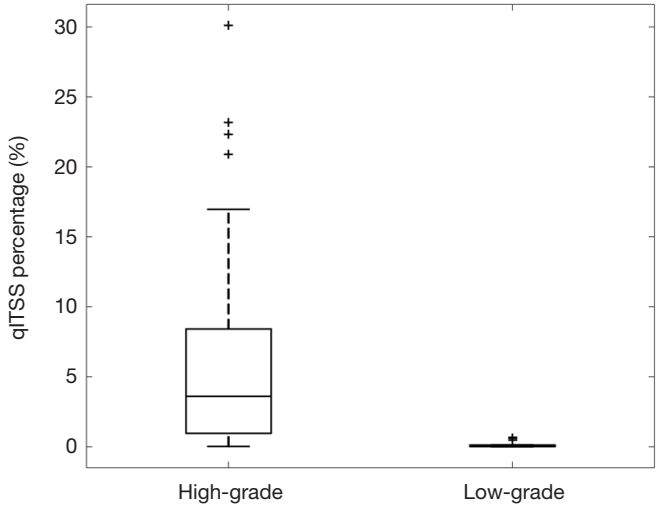

Figure 3 The box plot of qITSS percentage for high-grade and low-grade tumors with a NC threshold of 14. qITSS, quantitative intra-tumoral susceptibility signals; NC, normalized contrast.

qITSS.

In semi-quantitative ITSS evaluation methods proposed previously $(6,12)$, the presence of ITSS was identified as conglomerated dots or fine linear structures, and the total number counted. In our experience, however, the agglomeration of tense hypointensities at relatively large volumes, as shown in the example of Figure 4, might hinder the counting of ITSS number and underestimate tumor malignancy by those methods. In Figure 4, the conglomeration of hypointensity in pre-contrast SWI could be a result of tense distribution of hemorrhage, but according to the definition of ITSS grade, it would be assigned as grade 1 (1-5 dots or linear structures) or 2 (6-10 dots or linear structures). In comparison with qualitative or semi-quantitative ITSS evaluation, the method proposed in this study using an automatic segmentation approach exhibits the obvious advantage of objectivity. For example, the qITSS percentage of this relatively small tumor $(3.78 \mathrm{~mL})$ is $10.22 \%$ at a NC threshold of 14 , far beyond the distribution of the qITSS percentage of low-grade astrocytoma, ranging from 0 to $0.68 \%$. In addition, the calculation of signal contrast \{the numerator of Eq. [1]\} took the intensity of non-ITSS region in the tumor as reference, instead of MR signal from any other tissue (8), rendering it stable for scans with different scanning parameters or at different main field strengths.

Another advantage of qITSS is that the evaluation is based on an investigation of $3 \mathrm{D}$ volume, instead of a single slice that visually shows maximum frequency of ITSS within the tumor. The presence and frequency of ITSS could highly depend on the location and orientation of the slice 



Figure 4 Axial images of a 50-year female patient with glioblastoma (WHO grade IV). (A) CE-T1WI. (B) SWI. (C) SWI with the delineation of tumor (in green) and qITSS (in red). The conglomerated hypointensity in SWI, combined with relatively small size of tumor $(3.78 \mathrm{~mL})$, reflects a high percentage of qITSS (10.22\% at a NC threshold of 14). In this case, the determination of ITSS score, which could be classified into either ITSS grade 1 or 2, is challenging, leading to possible underestimation the tumor malignancy. WHO, World Health Organization; CE T1WI, contrast-enhanced T1-weighted image; SWI, susceptibility-weighted image; qITSS, quantitative intra-tumoral susceptibility signal; NC, normalized contrast; ITSS, intra-tumoral susceptibility signal.

because the distribution of hemorrhage or angiogenesis might not be uniform in the tumor and is often known to demonstrate substantial spatial heterogeneity. In Figure 5 as an instance, five distinct and equally-spaced slices are selected to demonstrate the CE T1WI (upper row) and SWI (middle and lower rows) of a 41-year female patient with low-grade astrocytoma (WHO grade II). The number of ITSS in these five cropped SWI could be less than 5 in the leftmost slice to more than 10 in the second from the right, which varies across slices to a great extent. According to the definition of semi-quantitative ITSS score $(7,12)$, this case would be classified to ITSS grade 3. However, the volume of the whole tumor and qITSS is 104.53 and $0.71 \mathrm{~mL}(\mathrm{NC}>14)$, respectively, only leading to a qITSS percentage of $0.68 \%$. Despite it is the very case with the highest percentage in the low-grade group of this study, the $0.68 \%$ percentage is less than the first quartile for the group of high-grade astrocytoma $(0.96 \%)$, still indicating low degree of invasion by paramagnetic substances.

Although comparatively objective with its precedents, our proposed method is subject to more technical improvements. First, the delineation of the tumor from slice to slice, which is manually drawn in this study, is laborintensive. In addition, the boundary of tumor is not always clear to define, especially for diffuse low-grade astrocytoma because of its infiltrative nature. Although with the help of deep learning algorithms, automatic segmentation of tumor from CE T1WI is possible (22), infiltration of active tumor beyond the boundary depicted on CE T1WI may further complicate the calculation of actual tumor volume and hence qITSS percentage. Both of the above issues are yet beyond the scope of this study.

Limitations of this study also include the relatively small sample size of low-grade astrocytoma $(n=15)$ and anaplastic astrocytoma $(n=5)$, which not only restricts the statistical power, but also precludes further investigation into each individual WHO grade. Oligodendroglioma, which constitutes $5-20 \%$ of all glial tumors (23), were also excluded from our study. While we did not include oligodendroglioma simply due to too few cases available ( $\mathrm{n}=3$ only), it should be noted that calcification occurs in approximately $80 \%$ of oligodendroglioma (24). Thus the high incidence of calcification, another piece of information that may be obtained from SWI (24), could potentially be used for differential diagnosis of oligodendroglioma from astrocytoma before surgical histology is available. Furthermore, the intra-group heterogeneity of the high-grade group, including glioblastoma (grade IV) and anaplastic astrocytoma (grade III), may hinder discrimination. For example, the endothelial vascular proliferation, which could appear as the ITSS in SWI, has been considered as one of the 

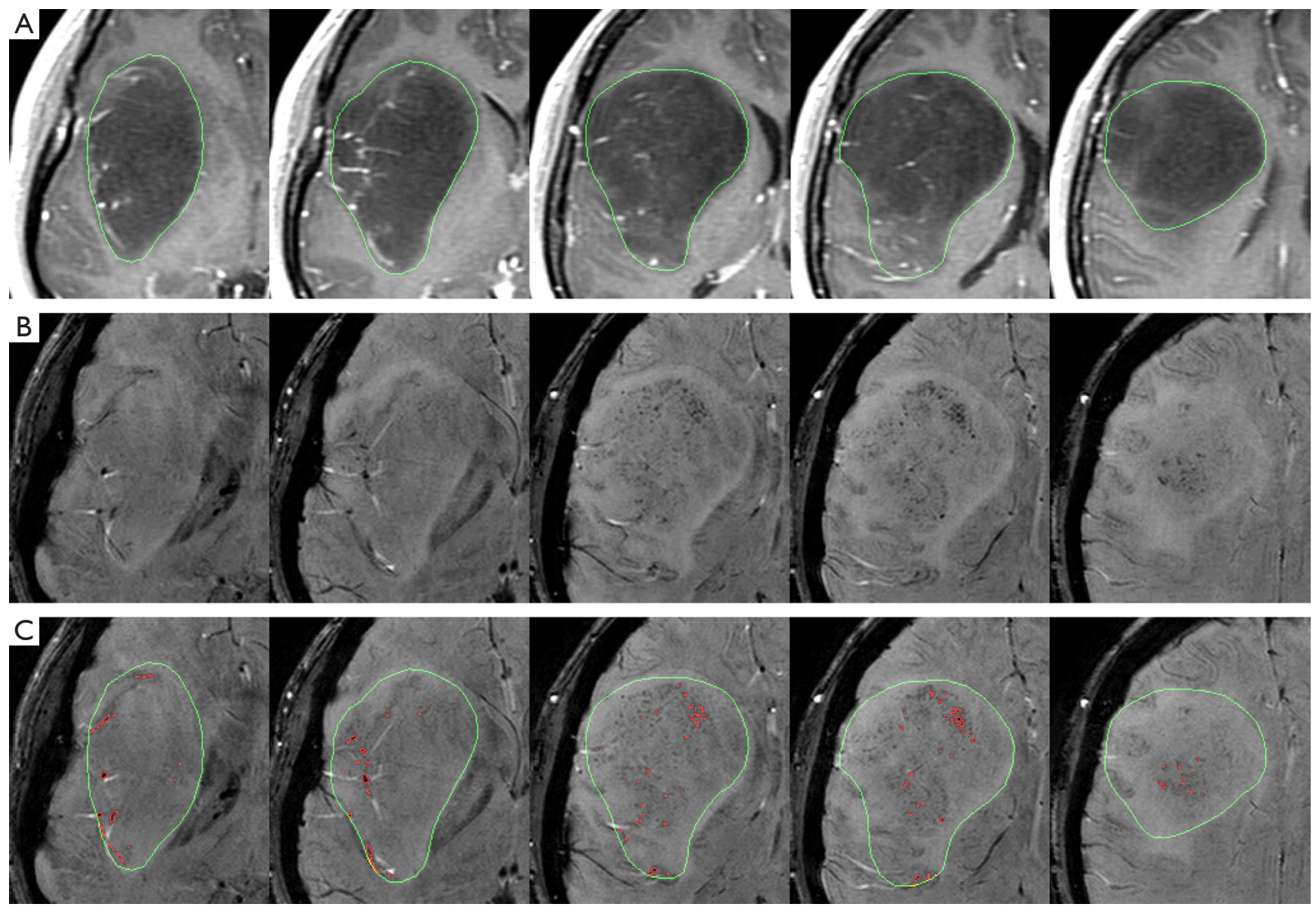

Figure 5 Five equally-spaced slices of a 41-year female with low-grade astrocytoma (WHO grade II). From top to bottom, the CE T1WI, SWI, and SWI with contours of the tumor (green) and qITSS (red) are shown. Each row is composed of five distinct axial slices in cropped view for more comprehensive observation of the tumor. Because the number of hypointense dots in a single slice, for example, the second from the right, could be more than 10, this case would be classified to ITSS grade 3 . However, the volume of the whole tumor and qITSS is $104.53 \mathrm{~mL}$ and $0.71 \mathrm{~mL}(\mathrm{NC}>14)$, only leading to a qITSS percentage of $0.68 \%$. WHO, World Health Organization; CE T1WI, contrastenhanced T1-weighted image; SWI, susceptibility-weighted image; qITSS, quantitative intra-tumoral susceptibility signal; ITSS, intratumoral susceptibility signal; NC, normalized contrast.

histological criteria for glioblastoma, but not for anaplastic astrocytoma, possibly leading to higher qITSS in more malignant tumors. Our results of ROC analysis with a NC threshold of 14 show that only four false negatives (high-grade tumors being classified into low grade) are found at the optimal operation point, and two of them are anaplastic astrocytoma, not to mention that the mean qITSS percentage of anaplastic astrocytoma $(0.42 \%)$ is much lower than that of glioblastoma (6.39\%). Future multi-center project may accelerate the collection of grade II and III astrocytoma, such that the potential value of SWI with qITSS quantification in clinical practice could be comprehensively investigated. Moreover, differentiation between increased vasculature and hemorrhage, both contributing to intratumoral dark susceptibility signal in SWI, was not attempted in our study. Tumor vasculature is known to be tortuous, with shape and size unpredictable just like in the case of hemorrhage. Whether the differentiation between vasculature and hemorrhage could help tumor grading cannot be answered by our study either, because we do not have pathology results with location registered to the original images for these patients. Besides, due to the absence of molecular genetic testing, such as IDH mutation, in participants earlier 
recruited, the revised 2016 WHO classification (25) is not suitable for this retrospective study. Nevertheless, we feel that this intrinsic limitation arising from the retrospective nature of our study should not preclude the importance of developing an objective method for ITSS quantification. Upon recruiting more patients for whom molecular genetic testing results are available, it would be interesting to investigate whether qITSS proposed in our study also helps differentiating the 2016 WHO classification categories. In addition, for institutes with limited resources in molecular testing, findings from our current qITSS study based on 2007 WHO classification would still be of practical value when only images are available. Lastly, the interference of susceptibility artifacts around air-tissue interfaces, which was carefully excluded before evaluation of ITSS, results in possible underestimation of lesion volume. These unsolved issues await further technical investigations.

Non-invasive imaging differentiation between low- and high-grade astrocytomas plays a critical role in planning patient management. In addition to SWI examined in this study, other MR imaging techniques that have been shown effective in tumor grading include perfusion-weighted imaging (12,15-17,26-30), diffusion-weighted imaging $(27,29,31,32)$, proton MR spectroscopy (26,27), and various combination of the above $(26,29)$. Perfusion-weighted MRI, by means of either dynamic susceptibility-contrast MRI (30) or arterial spin-labeling approaches (28), targets at tumor angiogenesis and/or tortuous vasculature. On the other hand, diffusion-weighted MRI probes the tissue micro-structure of the lesions, in particular cellularity (33). Proton MR spectroscopy further helps visualize the biochemical constituents from tumor metabolism (34), although the time-consuming nature may have precluded it from being included universally as a routine protocol. The distinct diagnostic information revealed by SWI, namely mainly venous vasculature and hemorrhage, should provide complementary value to the above MR imaging methods in terms of tumor grading. $(15,17)$ It would therefore be interesting to investigate whether the qITSS index proposed in this study could further enhance low- and high-grade astrocytoma differentiation when combined with perfusionand diffusion-weighted MR imaging.

\section{Conclusions}

In conclusion, the quantitative measurement of ITSS percentage proposed in this study is able to provide useful information in grading of astrocytoma.

\section{Acknowledgments}

Funding: The work was supported by the Ministry of Science and Technology research grants (MOST 101-2221-E-002013-MY3, MOST 104-2221-E-110-039, MOST 105-2221E-110-054, and MOST 107-2221-E-002-038-MY3) and Kaohsiung Veterans General Hospital (VGHKS107-160, VGHKS108-156, and VGHKS109-144).

\section{Footnote}

Conflicts of Interest: All authors have completed the ICMJE uniform disclosure form (available at https://dx.doi. org/10.21037/qims-21-58). Dr. HWC serves as an unpaid editorial board member of Quantitative Imaging in Medicine and Surgery, and Drs. TCC, YLC, WPS, HWC reported that they received grant supports from Ministry of Science and Technology (Taiwan) research grants (MOST 1042221-E-110-039 and MOST 105-2221-E-110-054); Dr. DHL reported that he received grant support from Kaohsiung Veterans General Hospital (VGHKS107-160) in Taiwan. The other authors have no conflicts of interest to declare.

Ethical Statement: The authors are accountable for all aspects of the work in ensuring that questions related to the accuracy or integrity of any part of the work are appropriately investigated and resolved. The study was conducted in accordance with the Declaration of Helsinki (as revised in 2013). The in vivo study was approved by the institutional review board of Kaohsiung Veterans General Hospital, and informed consents were obtained from all patients.

Open Access Statement: This is an Open Access article distributed in accordance with the Creative Commons Attribution-NonCommercial-NoDerivs 4.0 International License (CC BY-NC-ND 4.0), which permits the noncommercial replication and distribution of the article with the strict proviso that no changes or edits are made and the original work is properly cited (including links to both the formal publication through the relevant DOI and the license). See: https://creativecommons.org/licenses/by-nc-nd/4.0/.

\section{References}

1. Reichenbach JR, Venkatesan R, Schillinger DJ, Kido DK, Haacke EM. Small vessels in the human brain: MR 
venography with deoxyhemoglobin as an intrinsic contrast agent. Radiology 1997;204:272-7.

2. Haacke EM, Xu Y, Cheng YC, Reichenbach JR. Susceptibility weighted imaging (SWI). Magn Reson Med 2004;52:612-8.

3. Mittal S, Wu Z, Neelavalli J, Haacke EM. Susceptibilityweighted imaging: technical aspects and clinical applications, part 2. AJNR Am J Neuroradiol 2009;30:232-52.

4. Sehgal V, Delproposto Z, Haddar D, Haacke EM, Sloan AE, Zamorano LJ, Barger G, Hu J, Xu Y, Prabhakaran KP, Elangovan IR, Neelavalli J, Reichenbach JR. Susceptibilityweighted imaging to visualize blood products and improve tumor contrast in the study of brain masses. J Magn Reson Imaging 2006;24:41-51.

5. Pinker K, Noebauer-Huhmann IM, Stavrou I, Hoeftberger R, Szomolanyi P, Karanikas G, Weber M, Stadlbauer A, Knosp E, Friedrich K, Trattnig S. Highresolution contrast-enhanced, susceptibility-weighted MR imaging at $3 \mathrm{~T}$ in patients with brain tumors: correlation with positron-emission tomography and histopathologic findings. AJNR Am J Neuroradiol 2007;28:1280-6.

6. Kim HS, Jahng GH, Ryu CW, Kim SY. Added value and diagnostic performance of intratumoral susceptibility signals in the differential diagnosis of solitary enhancing brain lesions: preliminary study. AJNR Am J Neuroradiol 2009;30:1574-9.

7. Park SM, Kim HS, Jahng GH, Ryu CW, Kim SY. Combination of high-resolution susceptibility-weighted imaging and the apparent diffusion coefficient: added value to brain tumour imaging and clinical feasibility of noncontrast MRI at 3 T. Br J Radiol 2010;83:466-75.

8. Radbruch A, Graf M, Kramp L, Wiestler B, Floca R, Baumer P, Roethke M, Stieltjes B, Schlemmer HP, Heiland S, Bendszus M. Differentiation of brain metastases by percentagewise quantification of intratumoral-susceptibility-signals at 3Tesla. Eur J Radiol 2012;81:4064-8.

9. Radbruch A, Wiestler B, Kramp L, Lutz K, Baumer P, Weiler M, Roethke M, Sahm F, Schlemmer HP, Wick W, Heiland S, Bendszus M. Differentiation of glioblastoma and primary CNS lymphomas using susceptibility weighted imaging. Eur J Radiol 2013;82:552-6.

10. Kickingereder P, Wiestler B, Sahm F, Heiland S, Roethke M, Schlemmer HP, Wick W, Bendszus M, Radbruch A. Primary central nervous system lymphoma and atypical glioblastoma: multiparametric differentiation by using diffusion-, perfusion-, and susceptibility-weighted MR imaging. Radiology 2014;272:843-50.

11. Fu JH, Chuang TC, Chung HW, Chang HC, Lin HS, Hsu SS, Wang PC, Hsu SH, Pan HB, Lai PH. Discriminating pyogenic brain abscesses, necrotic glioblastomas, and necrotic metastatic brain tumors by means of susceptibility-weighted imaging. Eur Radiol 2015;25:1413-20.

12. Park MJ, Kim HS, Jahng GH, Ryu CW, Park SM, Kim SY. Semiquantitative assessment of intratumoral susceptibility signals using non-contrast-enhanced high-field highresolution susceptibility-weighted imaging in patients with gliomas: comparison with MR perfusion imaging. AJNR Am J Neuroradiol 2009;30:1402-8.

13. Li C, Ai B, Li Y, Qi H, Wu L. Susceptibility-weighted imaging in grading brain astrocytomas. Eur J Radiol 2010;75:e81-5.

14. Hori M, Mori H, Aoki S, Abe O, Masumoto T, Kunimatsu S, Ohtomo K, Kabasawa H, Shiraga N, Araki T. Threedimensional susceptibility-weighted imaging at $3 \mathrm{~T}$ using various image analysis methods in the estimation of grading intracranial gliomas. Magn Reson Imaging 2010;28:594-8.

15. Wang XC, Zhang H, Tan Y, Qin JB, Wu XF, Wang L, Zhang L. Combined value of susceptibility-weighted and perfusion-weighted imaging in assessing WHO grade for brain astrocytomas. J Magn Reson Imaging 2014;39:1569-74.

16. Li X, Zhu Y, Kang H, Zhang Y, Liang H, Wang S, Zhang W. Glioma grading by microvascular permeability parameters derived from dynamic contrast-enhanced MRI and intratumoral susceptibility signal on susceptibility weighted imaging. Cancer Imaging 2015;15:4.

17. Saini J, Gupta PK, Sahoo P, Singh A, Patir R, Ahlawat S, Beniwal M, Thennarasu K, Santosh V, Gupta RK. Differentiation of grade II/III and grade IV glioma by combining "T1 contrast-enhanced brain perfusion imaging" and susceptibility-weighted quantitative imaging. Neuroradiology 2018;60:43-50.

18. Louis DN, Ohgaki H, Wiestler OD, Cavenee WK, Burger PC, Jouvet A, Scheithauer BW, Kleihues P. The 2007 WHO classification of tumours of the central nervous system. Acta Neuropathol 2007;114:97-109.

19. Chang HC, Chuang TC, Chung HW, Lin HS, Lai PH, Weng MJ, Fu JH, Wang PC, Li SC, Pan HB. Multilayer appearance on contrast-enhanced susceptibility-weighted images on patients with brain abscesses: Possible origins and effects of postprocessing. J Magn Reson Imaging 2012;36:1353-61. 
20. Collignon A, Maes F, Delaere D, Vandermeulen D, Suetens P, Marchal G. Automated multi-modality image registration based on information theory. Proc Information Processing in Medical Imaging 1995. p. 263-74.

21. Otsu N. A threshold selection method from gray-level histograms. IEEE Trans Syst Man Cybern 1979;9:62-6.

22. Pereira S, Pinto A, Alves V, Silva CA. Brain Tumor Segmentation Using Convolutional Neural Networks in MRI Images. IEEE Trans Med Imaging 2016;35:1240-51.

23. Van den Bent MJ, Reni M, Gatta G, Vecht C. Oligodendroglioma. Crit Rev Oncol Hematol 2008;66:262-72.

24. Zulfiqar M, Dumrongpisutikul N, Intrapiromkul J, Yousem DM. Detection of intratumoral calcification in oligodendrogliomas by susceptibility-weighted MR imaging. AJNR Am J Neuroradiol 2012;33:858-64.

25. Louis DN, Perry A, Reifenberger G, von Deimling A, Figarella-Branger D, Cavenee WK, Ohgaki H, Wiestler OD, Kleihues P, Ellison DW. The 2016 World Health Organization Classification of Tumors of the Central Nervous System: a summary. Acta Neuropathol 2016;131:803-20.

26. Law M, Yang S, Wang H, Babb JS, Johnson G, Cha S, Knopp EA, Zagzag D. Glioma grading: sensitivity, specificity, and predictive values of perfusion MR imaging and proton MR spectroscopic imaging compared with conventional MR imaging. AJNR Am J Neuroradiol 2003;24:1989-98.

27. de Fatima Vasco Aragao M, Law M, Batista de Almeida D, Fatterpekar G, Delman B, Bader AS, Pelaez M, Fowkes M, Vieira de Mello R, Moraes Valenca M. Comparison of perfusion, diffusion, and MR spectroscopy between lowgrade enhancing pilocytic astrocytomas and high-grade astrocytomas. AJNR Am J Neuroradiol 2014;35:1495-502.

28. Furtner J, Schopf V, Schewzow K, Kasprian G, Weber M, Woitek R, Asenbaum U, Preusser M, Marosi C, Hainfellner JA, Widhalm G, Wolfsberger S, Prayer D. Arterial spin-labeling assessment of normalized vascular

Cite this article as: Chuang TC, Chen YL, Shui WP, Chung HW, Hsu SS, Lai PH. Intra-tumoral susceptibility signal: a post-processing technique for objective grading of astrocytoma with susceptibility-weighted imaging. Quant Imaging Med Surg 2022;12(1):558-567. doi: 10.21037/qims-21-58 intratumoral signal intensity as a predictor of histologic grade of astrocytic neoplasms. AJNR Am J Neuroradiol 2014;35:482-9.

29. Xiao HF, Chen ZY, Lou X, Wang YL, Gui QP, Wang Y, Shi KN, Zhou ZY, Zheng DD, Wang DJ, Ma L. Astrocytic tumour grading: a comparative study of threedimensional pseudocontinuous arterial spin labelling, dynamic susceptibility contrast-enhanced perfusionweighted imaging, and diffusion-weighted imaging. Eur Radiol 2015;25:3423-30.

30. You SH, Choi SH, Kim TM, Park CK, Park SH, Won JK, Kim IH, Lee ST, Choi HJ, Yoo RE, Kang KM, Yun TJ, Kim JH, Sohn CH. Differentiation of highgrade from low-grade astrocytoma: improvement in diagnostic accuracy and reliability of pharmacokinetic parameters from DCE MR imaging by using arterial input functions obtained from DSC MR imaging. Radiology 2018;286:981-91.

31. Murakami R, Hirai T, Kitajima M, Fukuoka H, Toya R, Nakamura H, Kuratsu J, Yamashita Y. Magnetic resonance imaging of pilocytic astrocytomas: usefulness of the minimum apparent diffusion coefficient (ADC) value for differentiation from high-grade gliomas. Acta Radiol 2008;49:462-7.

32. Chen Z, Ma L, Lou X, Zhou Z. Diagnostic value of minimum apparent diffusion coefficient values in prediction of neuroepithelial tumor grading. J Magn Reson Imaging 2010;31:1331-8.

33. Sugahara T, Korogi Y, Kochi M, Ikushima I, Shigematu Y, Hirai T, Okuda T, Liang L, Ge Y, Komohara Y, Ushio Y, Takahashi M. Usefulness of diffusion-weighted MRI with echo-planar technique in the evaluation of cellularity in gliomas. J Magn Reson Imaging 1999;9:53-60.

34. Alger JR, Frank JA, Bizzi A, Fulham MJ, DeSouza BX, Duhaney MO, Inscoe SW, Black JL, van Zijl PC, Moonen CT. Metabolism of human gliomas: assessment with H-1 MR spectroscopy and F-18 fluorodeoxyglucose PET. Radiology 1990;177:633-41. 